\title{
Deteç̧ão de Estímulos Concêntricos Mesópicos em Crianças Surdas e Ouvintes ${ }^{1}$
}

\author{
Natanael Antonio dos Santos ${ }^{2}$ \\ Universidade Federal da Paraíba \\ Liana Chaves Mendes \\ Universidade Federal de Pernambuco \\ Valtenice de Cássia Rodrigues de Matos França \\ Universidade de São Paulo \\ Aline Mendes Lacerda \\ Universidade de São Paulo
}

\begin{abstract}
RESUMO - O objetivo deste trabalho foi medir curvas de sensibilidade ao contraste de 10 crianças ouvintes e de 10 crianças com surdez pré-lingual, de 7 a 12 anos, utilizando frequências radiais circularmente concêntricas (FSCr) de 0,25-2,0 cpg em níveis baixos de luminância $\left(0,7 \mathrm{~cd} / \mathrm{m}^{2}\right)$. Todos os participantes apresentavam acuidade visual normal e estavam livres de doenças oculares identificáveis. A FSCr foi medida com o método psicofísico da escolha forçada. Os resultados mostraram sensibilidade máxima na faixa de frequência radial de 0,25 cpg para os dois grupos. Os resultados mostraram ainda diferenças significantes entre as curvas de FSCr de crianças ouvintes e de crianças com surdez pré-lingual. Isto é, as crianças ouvintes precisaram de menos contraste do que as crianças surdas para detectar as frequências radiais. Esses resultados sugerem que, em níveis baixos de luminância, a FSCr das crianças ouvintes foi melhor do que a das crianças com surdez pré-lingual.
\end{abstract}

Palavras-chave: percepção visual de contraste; plasticidade; crianças surdas; frequência radial; método da escolha forçada.

\section{Detection of Mesopic Concentric Stimuli in Deaf and Hearing Children}

\begin{abstract}
The aim of this work was to measure contrast sensitivity curves in 10 hearing children and 10 children with prelingual deafness (from 7 to 12 years old), using concentric circular patterns with radial frequencies (rCSF) of 0.25-2.0 cpd at low levels of luminance $\left(0.7 \mathrm{~cd} / \mathrm{m}^{2}\right)$. All participants were free from identifiable ocular disease and had normal visual acuity. The rCSF was measured with the psychophysical forced-choice method. The results showed maximum sensitivity in the frequency range of $0.25 \mathrm{cpd}$ for the two groups. The results showed yet significant differences between the rCSF of deaf and hearing children. That is, hearing children needed less contrast than deaf children to detect radial frequencies. These results suggest that at low levels of luminance the rCSF of hearing children was better than the rCSF of children with prelingual deafness.
\end{abstract}

Keywords: contrast visual perception; plasticity; deaf children; radial frequency; forced-choice method.

Muitas pesquisas, desde os estudos pioneiros de Hubel e Wiesel na década de $60(1962,1965,1968)$, relatam que a privação sensorial parcial ou total de um sistema sensitivo pode alterar a resposta do sistema privado de estimulação e/ou de outros sistemas sensoriais. Isso acontece porque os sistemas sensoriais privados de estimulação tentam naturalmente se reorganizar em função das alterações sensoriais sofridas e das condições ambientais. Porém, poucos estudos têm utilizado o limiar visual de contraste para avaliar e caracterizar os efeitos da plasticidade neural (capacidade que o encéfalo possui de se reorganizar) decorrente da privação de estímulos auditivos sobre as vias e funções visuais.

1 Pesquisa financiada pelo $\mathrm{CNPq} / \mathrm{MCT}$ por meio de Bolsa de Mestrado e Bolsa de Produtividade em Pesquisa (processo: 304716/2008-3). Trabalho apresentado na XXI Reunião Anual da Federação de Sociedades de Biologia Experimental (FeSBE).

2 Endereço para correspondência: Rua Manoel Belarmino de Macedo, $\mathrm{n}^{\circ}$ 355, $\mathrm{Apt}^{\mathrm{0}}$ 402. Ed. Camburiú, Jardim Cidade Universitária. João Pessoa, PB. CEP 58052-290. Telefone: (83) 3235-4439, Fax: (83) 3216-7064. E-mail: natanael_labv@yahoo.com.br.
Em sua maioria, os estudos com humanos têm se limitado a investigar as habilidades visuais cognitivas relacionadas à surdez, como por exemplo, o processo de atenção para estímulos em movimento em diferentes condições (Bavelier, Dye \& Hauser, 2006; Bavelier \& cols., 2000; Bosworth \& Dobkins, 2002; Neville \& Lawson, 1987; Proksch \& Bavelier, 2002; Rettenbach, Diller \& Sireteanu, 1999). Inclusive, a maior parte dessas pesquisas relata que os voluntários com privação auditiva apresentam maiores habilidades relacionadas à atenção visual na periferia do campo visual do que as pessoas ouvintes sem prejuízos.

Atualmente, a curva de sensibilidade ao contraste (FSC) tem sido muito utilizada para avaliar e diagnosticar alterações neuropatológicas nas vias sensoriais visuais e no sistema nervoso, produzidas por vários transtornos ou doenças, como por exemplo: esquizofrenia (Slaghuis \& Thompson, 2003), depressão (Cavalcanti \& Santos, 2005), síndrome de Down (Suttle \& Turner, 2004), ambliopia (Polat, Sagi \& Norcia, 1997), catarata (Elliott \& Situ, 1998), glaucoma (Silva \& Rodrigues, 2002), intoxicação por mercúrio (Ventura \& cols., 2005) e doenças de Alzheimer e de Parkinson (Akutsu \& 

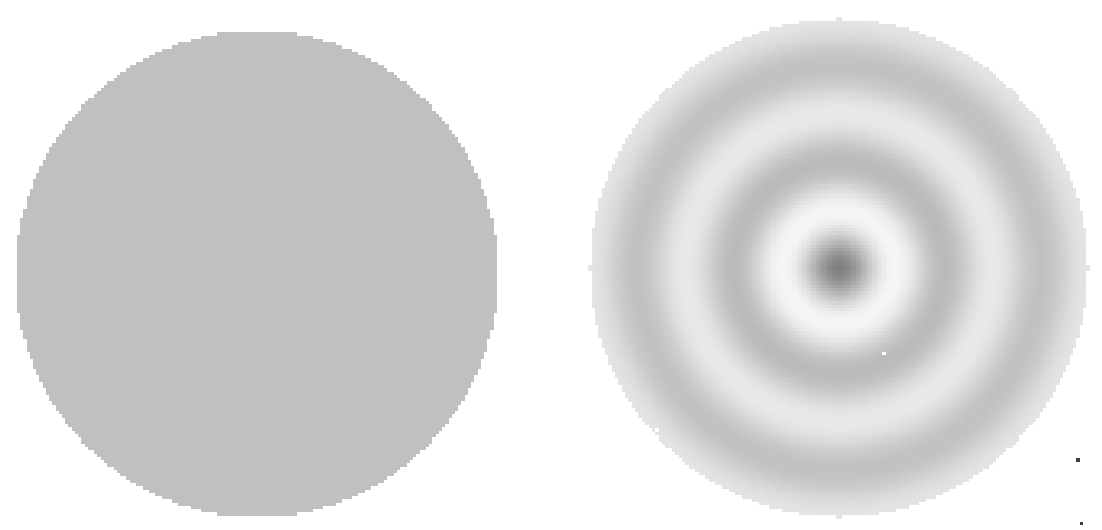

Figura 1. Exemplo de um par de estímulos, à esquerda estímulo neutro e à direta a frequência radial de 1,0 cpg. Estímulos originalmente calibrados para serem vistos a 150 cm de distância.

Legge, 1995; Bour \& Apkarian, 1996; Elliott \& Situ, 1998; Polat \& cols., 1997; Vleugels, van Nunen, Lafosse, Ketelaer \& Vandenbussche, 1998). A ideia fundamental sobre a utilização da FSC é que a redução ou a elevação do limiar sensório seja um sinal de alterações nos mecanismos ou nas vias sensórias que processam contraste.

A FSC é a recíproca da quantidade mínima de contraste necessária para detectar um objeto qualquer ou uma grade senoidal de uma determinada frequência espacial (Cornsweet, 1970). Assim, na medição da sensibilidade ao contraste, procura-se estabelecer, experimentalmente, o valor mínimo de contraste que o sistema visual precisa para detectar um determinado estímulo ou frequência espacial. Frequência espacial é o número de ciclos por unidade de espaço, convencionalmente denominada de ciclos por grau de ângulo visual, cpg (Schwartz, 2004). Por exemplo, uma frequência espacial de 2,0 cpg tem duas listras claras e duas escuras em um determinado espaço.

Pesquisas relacionando a FSC à surdez em adultos são raras e inexistentes em crianças. No único estudo encontrado na literatura, Finney e Dobkins (2001) utilizaram a FSC para avaliar os efeitos da surdez na percepção visual em adultos. Eles mediram com o método psicofísico da escada os limiares de contraste para 34 adultos (13 surdos, 14 ouvintes e sete ouvintes descendentes de surdos que sabiam a linguagem de sinais), utilizando grades senoidais horizontais em movimento e com luminância média de $28 \mathrm{~cd} / \mathrm{m}^{2}$. Os resultados não indicaram alterações no limiar sensório dos adultos surdos, ouvintes e ouvintes que sabiam fluentemente a linguagem de sinais.

Neste sentido, o presente estudo teve como objetivo determinar e comparar a FSC de crianças ouvintes e de crianças com surdez pré-lingual de 7 a 12 anos. A proposição geral era verificar se a privação sensorial do sistema auditivo durante o desenvolvimento alteraria a curva de limiar sensório ou a percepção visual de crianças surdas. As medidas de limiar ou de sensibilidade ao contraste para estímulos radiais circularmente simétricos estáticos (FSCr), com luminância média de $0,7 \mathrm{~cd} / \mathrm{m}^{2}$, foram realizadas com o método psicofísico da escolha forçada.

\section{Método}

\section{Participantes}

Participaram do experimento 20 crianças de ambos os sexos com idades entre 7 e 12 anos, 10 com audição normal $(\mathrm{M}=11,3 ; \mathrm{DP}=1,79)$ e 10 com surdez pré-lingual $(\mathrm{M}=$ 11,$5 ; \mathrm{DP}=1,78$ ). Todas apresentavam acuidade visual normal ou corrigida e não tinham histórico de patologia ocular ou neurológica. A acuidade visual foi medida com a cartela de optotipos "E” de Rasquin.

As crianças surdas apresentavam surdez pré-lingual ${ }^{3}$ (surdez adquirida em um período que antecede a linguagem falada) bilateral, de grau severo e profundo (perda auditiva $\geq 80 \mathrm{~dB}$ ). Dessas, oito apresentavam surdez congênita (três decorrentes de causas desconhecidas e cinco de rubéola materna) e duas apresentavam surdez adquirida (uma decorrente de meningite e a outra do efeito de medicamentos). Todas as crianças surdas sabiam LIBRAS (Língua Brasileira de Sinais) e foram encaminhadas ao laboratório pelo Centro SUVAG (Sistema Universal Verbotonal da Audição Guberina) da Paraíba e pelo CEPES (Centro de Educação Permanente para Surdos).

A participação na pesquisa ocorreu mediante a assinatura de termo de consentimento livre e esclarecido pelos responsáveis, conforme a Resolução no 196/96 do Conselho Nacional de Saúde (Ministério da Saúde), que trata das diretrizes e normas de pesquisas envolvendo seres humanos. O projeto foi aprovado pelo Comitê de Ética do Centro de Ciências da Saúde da Universidade Federal da Paraíba.

\section{Equipamentos e estímulos visuais}

Os estímulos foram gerados num monitor de vídeo monocromático CLINTON MEDICAL de 21 polegadas, tela plana, digital, de alta resolução, controlado por um

3 Outras informações sobre a classificação da surdez (e.g., pré-lingual) podem ser encontradas na literatura (Cordes \& Wszolek, 2003; Oliveira, Castro \& Ribeiro, 2002). 
microcomputador. A luminância média da tela foi de 0,7 $\mathrm{cd} / \mathrm{m}^{2}$ ajustada por um fotômetro do tipo SPOT METTER, com precisão de um grau, ASAHI PENTAX. A luminância mínima da tela foi de $0,5 \mathrm{~cd} / \mathrm{m}^{2}$ e a máxima de $0,9 \mathrm{~cd} / \mathrm{m}^{2}$. O ambiente do laboratório, onde os dados foram coletados, era cinza, para melhor controle da luminância.

Foram utilizados estímulos circularmente simétricos, acromáticos e estáticos com frequências radiais de 0,25 , 0,5, 1,0 e 2,0 ciclos por grau de ângulo visual (cpg) e um estímulo neutro homogêneo com luminância média de 0,7 $\mathrm{cd} / \mathrm{m}^{2}$ (ver exemplos na Figura 1). Todos os estímulos tinham um diâmetro de aproximadamente 7,2 graus de ângulo visual a $150 \mathrm{~cm}$ de distância. Informações sobre estímulos radiais circularmente concêntricos podem ser encontradas na literatura (Kelly, 1960; Santos, Oliveira, Nogueira \& Simas, 2006; Santos, Simas \& Nogueira, 2004; Simas, Nogueira \& Santos, 2005).

\section{Procedimento}

As medidas de limiar de contraste foram realizadas com método psicofísico da escolha forçada (França, Santos \& Mendes, 2006; Santos, Nogueira \& Simas, 2005; Santos \& cols., 2006; Santos, Simas \& Nogueira, 2007). Esse método se baseia no cálculo da probabilidade de acertos consecutivos por parte do voluntário. Ou seja, em cerca de 50 oportunidades de escolhas entre os dois estímulos (estímulo de teste e estímulo neutro), a frequência radial (estímulo de teste) foi percebida, por exemplo, em 79\% das vezes pelo voluntário. O procedimento para medir o limiar para cada frequência consistiu na apresentação sucessiva simples de pares de estímulos, e um dos estímulos de cada par era o estímulo teste, que deveria ser identificado pelo participante (a Figura 1 mostra um exemplo de um par de estímulos). O critério adotado para variar o contraste da frequência radial de teste era o de três acertos consecutivos para decrescer uma unidade e um erro para acrescer da mesma unidade (0,08\%). A ordem de apresentação dos estímulos era aleatória e controlada pelo programa. Cada estímulo era apresentado por $2 \mathrm{~s}$, com intervalo entre estímulos de $1 \mathrm{~s}$ e com intervalo entre tentativas, ou par de estímulos, de $3 \mathrm{~s}$. A duração de cada sessão experimental variou entre 5 e 10 minutos, dependendo de quando os erros e acertos do participante proporcionaram um total de seis reversões (ou seis valores de contraste, três máximos e três mínimos), conforme requerido para o final automático da mesma.

Cada uma das frequências radiais da curva de FSCr foi estimada pelo menos duas vezes (duas sessões experimentais), em dias diferentes, por cada um dos participantes. Em média, 20 curvas foram medidas para cada grupo de voluntários, totalizando 40 curvas. Os limiares foram medidos à distância de $150 \mathrm{~cm}$, com visão binocular e pupila natural.

Todos os participantes receberam a seguinte instrução:

Será apresentada uma sequência de pares de estímulos, um com círculos claros e escuros e outro totalmente cinza. Você deverá escolher sempre o estímulo que contém os círculos, pressionando o botão do lado esquerdo (botão número 1) do mouse quando o estímulo com círculos for apresentado primeiro, e o botão do lado direito (botão número 2), quando este for apresentado em segundo lugar (após o estímulo cinza).

O mouse era sinalizado com os números " 1 " (botão esquerdo) e "2" (botão direito). É importante mencionar que cada sessão foi iniciada com o estímulo teste com contraste em nível supralimiar e que os experimentos só começaram quando o experimentador certificou-se que os participantes entenderam e responderam conforme as instruções. As instruções para os participantes surdos foram dadas em LIBRAS.

\section{Resultados}

Após cada sessão, o programa produziu uma folha de resultados com a situação experimental e os seis valores de contraste obtidos pelas reversões. Os valores de contraste obtidos para cada frequência foram agrupados em planilhas por condição (surdez e ouvinte), e a grande média foi utilizada como estimativa da sensibilidade ao contraste em função de cada frequência radial testada.

A Figura 2 mostra a FSCr de crianças surdas e ouvintes para frequências radiais de 0,25, 0,5, 1,0 e 2,0 cpg. A sensibilidade ao contraste (FSCr) é o inverso do limiar de contraste (1/FSCr). Logo, quanto menor o limiar de contraste, maior a sensibilidade do sistema visual humano e vice-versa. Assim, os menores valores de limiares correspondem aos maiores valores de sensibilidade ao contraste. As barras verticais em cada uma das curvas de FSCr indicam os erros padrões das médias.

As curvas de sensibilidade ao contraste apresentam perfis semelhantes, tendo a sensibilidade máxima ocorrido na frequência radial de 0,25 cpg para os dois grupos. Nessa frequência, as crianças surdas foram 1,4 vez menos sensíveis do que as ouvintes. Já nas frequências de $0,5,1,0$ e 2,0 cpg, as crianças ouvintes precisaram, em média, de 1,2, 1,1 e 1,1 menos contraste do que as crianças surdas, respectivamente.

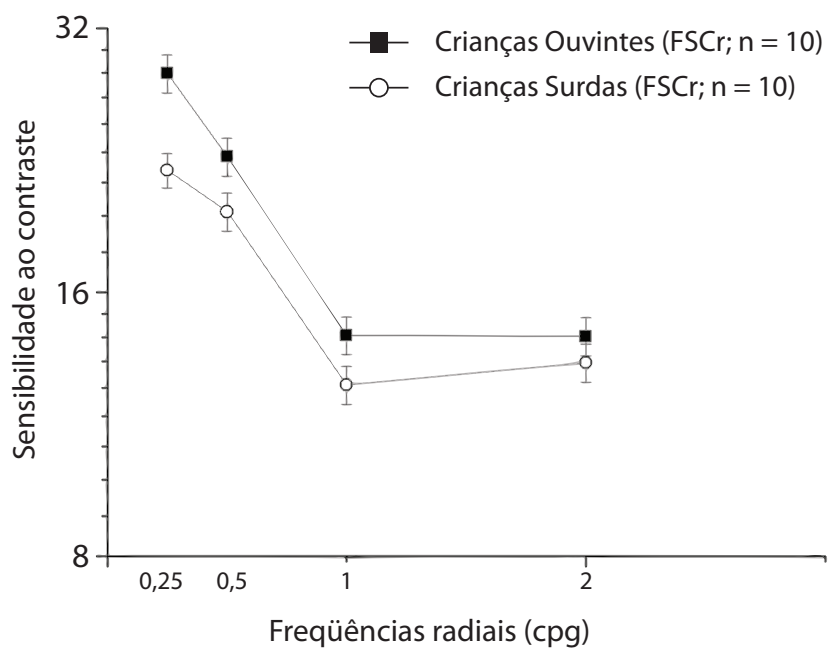

Figura 2. Curvas de sensibilidade ao contraste de crianças ouvintes e de crianças surdas de 7 a 12 anos para frequências radiais (FSCr). As linhas verticais mostram o erro padrão da média para cada frequência $(0,25 ; 0,5 ; 1,0$ e 2,0 cpg). 
A ANOVA para medidas repetidas revelou diferenças significativas entre as curvas de FSCr (média geral) das crianças surdas e das crianças com a audição normal [ $\mathrm{F}_{(1,238)}$ = 11,70; $\mathrm{p}<0,001]$. As análises com o teste post-hoc Tukey HSD não mostraram diferenças significativas na comparação entre os dois grupos para as frequências radiais de $0,25,0,5$, $1,0$ e 2,0 cpg ( $p>0,05)$.

No geral, esses resultados mostram diferenças significantes entre as médias gerais dos dois grupos, indicando que as crianças ouvintes podem ser mais sensíveis do que as crianças surdas para processar padrões circularmente simétricos com frequências radiais em condições mesópicas.

\section{Discussão}

O objetivo deste estudo foi determinar e comparar a FSCr de crianças ouvintes e de crianças com surdez pré-lingual na faixa etária de 7 a 12 anos. Mais especificamente, foi verificado se a plasticidade sensorial decorrente da surdez alteraria o limiar sensório visual ou a sensibilidade ao contraste para estímulos radiais circularmente simétricos em níveis baixos de luminância.

Os resultados mostraram diferenças estatísticas entre a sensibilidade e o contraste médio das crianças surdas e das crianças ouvintes para frequências radiais em condições mesópicas $\left(0,7 \mathrm{~cd} / \mathrm{m}^{2}\right)$, demonstrando que as crianças ouvintes apresentaram limiares de contraste médios aproximadamente 1,2 maiores que as crianças surdas. Entretanto, o teste post-hoc Tukey não revelou diferença significativa entre as frequências $(p>0,05)$. Isso significa que, embora o padrão de comportamento da FSC indique diferença entre os dois grupos, os resultados não são conclusivos, pois o teste posthoc não revelou diferenças entre os dois grupos em nenhuma das frequências testadas.

Algumas contradições nas pesquisas que relacionam a surdez com a percepção visual geralmente são atribuídas às metodologias e aos critérios de amostragem utilizados (Bavelier \& cols., 2006; Rettenbach \& cols., 1999; Stevens $\&$ Neville, 2006). No presente estudo, foi utilizado o método psicofísico da escolha forçada, um paradigma bem estabelecido na literatura, que minimiza os efeitos do critério de escolha de resposta dos participantes (Santos \& cols., 2004, 2006, 2007; Simas \& cols., 2005; Wetherill \& Levitt, 1965). Em estudos recentes realizados no Laboratório de Percepção, Neurociências e Comportamento da Universidade Federal da Paraíba (LPNeC-UFPB), foi demonstrado que o método psicofísico da escolha forçada, geralmente empregado em pesquisas com adultos, pode ser utilizado para medir a função de sensibilidade ao contraste de crianças a partir dos 4 anos de idade (França \& cols., 2006; Santos \& França, 2006).

Em relação à amostra, estudos desta natureza, com limiar sensório, método psicofísico e medidas repetidas, como é peculiar às pesquisas em percepção visual, envolvem um número pequeno de voluntários em cada condição e o critério de amostragem não é crítico, uma vez que se avaliam aspectos mais automáticos da percepção ou processos sensoriais (Finney \& Dobkins, 2001; França \& cols., 2006; Santos \& França, 2006).
Outra questão referente à amostra é que a idade de aparecimento e a etiologia da surdez podem alterar de forma diferente a percepção visual (FSCr) das crianças surdas. Isso pode explicar, em parte, porque o teste post-hoc Tukey não demonstrou diferença significativa na comparação entre as frequências testadas, uma vez que algumas crianças surdas participantes apresentavam surdez congênita e outras apresentavam surdez adquirida, decorrentes de diversas causas.

A redução de qualquer natureza na FSCr das crianças surdas não era necessariamente o esperado. Primeiro, porque os estudos relacionando o processo de atenção para estímulos em movimento em diferentes condições, relatam que os voluntários com privação auditiva apresentam maiores habilidades relacionadas à atenção visual na periferia do campo visual do que as pessoas ouvintes (Bavelier \& cols., 2006; Bavelier \& cols., 2000; Bosworth \& Dobkins, 2002; Neville \& Lawson, 1987; Proksch \& Bavelier, 2002; Rettenbach \& cols., 1999). Segundo, porque o estudo de Finney e Dobkins (2001) não encontrou diferenças entre os limiares de contraste de adultos ouvintes e surdos. Entretanto, não é possível fazer uma comparação direta entre os resultados desta pesquisa com as pesquisas que atentam para estímulos móveis, pois tratam de processos e construtos distintos do ponto de vista teórico e metodológico. Assim, não é possível comparar diretamente os resultados deste trabalho com crianças com os resultados da pesquisa com adultos de Finney e Dobkins, pois o nível de desenvolvimento sensorial é diferente entre adultos e crianças. Além disto, esses autores utilizaram condições metodológicas diferentes das utilizadas na presente pesquisa. Por exemplo, eles mediram o limiar de contraste de adultos surdos para grade senoidal horizontal móvel em condições fotópicas (luminância média de $28 \mathrm{~cd} / \mathrm{m}^{2}$ ). Já este trabalho mediu o limiar de contraste de crianças surdas para frequências radiais estáticas e condições mesópicas $\left(0,7 \mathrm{~cd} / \mathrm{m}^{2}\right)$.

Uma questão que precisa ser avaliada é se a surdez interage de forma diferente com as vias visuais. Isso porque a literatura tem apontado que o processamento visual da forma e de contraste envolve, pelo menos, dois sistemas: (a) a via visual parvocelular, que é especializada no processamento de frequências espaciais médias e altas ou detalhes finos, e opera em níveis altos ou fotópicos de luminância; e (b) a via visual magnocelular, que é especializada no processamento de frequências espaciais baixas e opera em níveis baixos ou escotópicos de luminância (Banks \& Bennett, 1988; Benedek, Benedek, Kéri, \& Janáky, 2003; Brown, Dobson \& Maier, 1987; Ellemberg, Lewis, Maurer \& Brent, 2000; Hickey, 1977; Kiorpes \& Movshon, 1998; Wilson, 1988; Yuodelis \& Hendrickson, 1986). Assim, pode ser que os efeitos da surdez interajam de forma distinta com as vias ou canais (grupos de neurônios) que processam estímulos em condições de luminâncias diferentes.

\section{Considerações Finais}

Esses resultados demonstram que o sistema visual de crianças surdas e ouvintes possui habilidades diferentes para detectar padrões radiais circularmente simétricos em condições mesópicas. Pois, a comparação entre a FSCr de crianças 
surdas e de crianças ouvintes demonstrou alterações na percepção visual das crianças surdas, que apresentaram menor sensibilidade ao contraste quando comparadas às crianças com audição normal. As alterações encontradas, por sua vez, podem estar relacionadas à plasticidade decorrente da surdez. Porém, em termos gerais, é cedo para afirmar que a surdez afeta o limiar sensório de contraste (FSCr) ou os mecanismos que processam bandas de frequências radiais mesópicas em crianças. Os resultados apresentados neste estudo são iniciais e novas pesquisas precisam ser realizadas para aprofundar e produzir novos dados empíricos com luminâncias, estímulos e populações diferentes. Só assim, será possível discutir de maneira mais sistemática as possíveis alterações na percepção visual relacionadas à surdez. É nessa perspectiva que o nosso laboratório prosseguirá com as investigações.

\section{Referências}

Akutsu, H., \& Legge, G. E. (1995). Discrimination of compound gratings: Spatial-frequency channels or local features? Vision Research, 35, 2685-2695.

Banks, M. S., \& Bennett, P. J. (1988). Optical and photoreceptor immaturities limit the spatial and chromatic vision of human neonates. Journal of the Optical Society of America A, 5, 20592079.

Bavelier, D., Dye, M. W. G., \& Hauser, P. C. (2006). Do deaf individuals see better? Trends in Cognitive Sciences, 10, 391-430.

Bavelier, D., Tomann, A., Hutton, C., Mitchell, T., Corina, D., Liu, G., \& Neville, H. (2000). Visual attention to the periphery is enhanced in congenitally deaf individuals. The Journal of Neuroscience, 20, 1-6.

Benedek, G., Benedek, K., Kéri, S., \& Janáky, M. (2003). The scotopic low-frequency spatial contrast sensitivity develops in children between the ages of 5 and 14 years. Neuroscience Letters, 345, 161-164.

Bosworth, R. G., \& Dobkins, K. R. (2002). Visual field asymmetries for motion processing in deaf and hearing signers. Brain and Cognition, 49, 170-181.

Bour, L. J., \& Apkarian, P. (1996). Selective broad-band spatial frequency loss in contrast sensitivity functions. Investigative Ophthalmology \& Visual Science, 37, 2475-2484.

Brown, A. M., Dobson, V., \& Maier, J. (1987). Visual acuity of human infants at scotopic, mesopic, and photopic luminances. Vision Research, 27, 1845-1858.

Cavalcanti, A. M., \& Santos, N. A. (2005). The effects of depression on contrast visual perception in humans: Preliminary results. Revista Brasileira de Terapias Cognitivas, 1, 21-27.

Cordes, M., \& Wszolek, Z. K. (2003). Deafness and cerebral plasticity. The Journal of Nuclear Medicine, 44, 1440-1442.

Cornsweet, T. N. (1970). Vision perception. New York: Academy Press.

Ellemberg, D., Lewis, T. L., Maurer, D., \& Brent, H. P. (2000). Influence of monocular deprivation during infancy on the later development of spatial and temporal vision. Vision Research, 40, 3283-3295.

Elliott, D. B., \& Situ, P. (1998). Visual acuity versus letter contrast sensitivity in early cataract. Vision Research, 38, 20472052.
Finney, E. M., \& Dobkins, K. R. (2001). Visual contrast sensitivity in deaf versus hearing populations: Exploring the perceptual consequences of auditory deprivation and experience with a visual language. Cognitive Brain Research, 11, 171-183.

França, V. C. R. M., Santos, N. A., \& Mendes, L. C. (2006). Sensibilidade ao contraste em crianças pré-escolares com método psicofísico. Psicologia: Teoria e Pesquisa, 22, 335-338.

Hickey, T. L. (1977). Postnatal development of the human lateral geniculate nucleus: Relationship to a critical period for the visual system. Science, 198, 836-838.

Hubel, D. H., \& Wiesel, T. N. (1962). Receptive fields, binocular interaction and functional architecture in the cat's visual cortex. Journal of Physiology, 160, 106-154.

Hubel, D. H., \& Wiesel, T. N. (1965). Binocular interaction in striate cortex of kittens reared with artificial squint. Journal of Neurophysiology, 28, 1041-1059.

Hubel, D. H., \& Wiesel, T. N. (1968). Receptive fields and functional architecture of monkey striate cortex. Journal of Physiology, 195, 215-243.

Kelly, D. H. (1960). J0 stimulus patterns for vision research. Journal of the Optical Society of America, 50, 1115-1116.

Kiorpes, L., \& Movshon, J. A. (1998). Peripheral and central factors limiting the development of contrast sensitivity in macaque monkeys. Vision Research, 38, 61-70.

Neville H. J., \& Lawson, D. (1987). Attention to central and peripheral visual space in a movement detection task: An eventrelated potential and behavioral study. II. Congenitally deaf adults. Brain Research, 405, 268-283.

Oliveira, P., Castro, F., \& Ribeiro, A. (2002). Surdez infantil. Revista Brasileira de Otorrinolaringologia, 68, 417-423.

Polat, U., Sagi, D., \& Norcia, A. M. (1997). Abnormal longrange spatial interactions in amblyopia. Vision Research, 37, 737-744.

Proksch, J., \& Bavelier, D. (2002). Changes in the spatial distribution of visual attention after early deafness. Journal of Cognitive Neuroscience, 14, 687-701.

Rettenbach, R., Diller, G., \& Sireteanu, R. (1999). Do deaf people see better? Texture segmentation and visual search compensate in adult but not in juvenile subjects. Journal of Cognitive Neuroscience, 11, 560-575.

Santos, N. A., \& França, V. C. R. M. (2006). Desenvolvimento da sensibilidade ao contraste para frequências espaciais em crianças. Psicologia em Estudo, 11, 599-605.

Santos, N. A., Nogueira, R. M. T. B. L., \& Simas, M. L. B. (2005). Processamento visual da forma: Evidências para canais múltiplos de frequências angulares em humanos. Psicologia: Reflexão e Crítica, 18, 98-103.

Santos, N. A., Oliveira, A. B., Nogueira, R. M. T. B. L., \& Simas, M. L. B. (2006). Mesopic radial frequency contrast sensitivity function for young and older adults. Brazilian Journal of Medical and Biological Research, 39, 791-794.

Santos, N. A., Simas, M. L. B., \& Nogueira, R. M. T. B. L. (2004). Comparison of angular frequency contrast sensitivity in young and older adults. Brazilian Journal of Medical and Biological Research, 37, 375-378.

Santos, N. A., Simas, M. L. B., \& Nogueira, R. M. T. B. L. (2007). Percepção visual de contraste em humanos: Evidência psicofísica para canais de frequência angular alta. Psicologia: Reflexão e Crítica, 20 , 559-567. 
Schwartz, S. H. (2004). Visual perception: A clinical orientation ( $3^{\mathrm{a}}$ ed.). New York: McGraw Hill.

Silva, A. C., \& Rodrigues, M. de L. V. (2002). Importância do estudo da função da sensibilidade ao contraste no glaucoma. Revista de Medicina, 35, 497-504.

Simas, M. L. B., Nogueira, R. M. T. B. L., \& Santos, N. A. (2005). Radial frequency stimuli and sine-wave gratings seem to be processed by distinct contrast brain mechanisms. Brazilian Journal of Medical and Biological Research, 38, 439-430.

Slaghuis, W. L., \& Thompson, A. K. (2003). The effect of peripheral visual motion on focal contrast sensitivity in positiveand negative-symptom schizophrenia. Neurophsychologia, 4, 968-980.

Stevens, C., \& Neville, H. (2006). Neuroplasticity as a doubleedged sword: Deaf enhancements and dyslexic deficits in motion processing. Journal of Cognitive Neuroscience, 18, 701-714.

Suttle, C. M., \& Turner, A. M. (2004). Transient pattern visual evoked potentials in children with Down`s syndrome. Ophthalmic and Physiological Optics, 24, 91-99.

Ventura, D. F, Simões, A. L., Tomaz, S., Costa, M. F., Lago, M., Costa, M. T. V., Canto-Pereira, L. H. M., de Souza, J. M., Faria M. A. M., \& Silveira, L. C. L. (2005). Colour vision and contrast sensitivity losses of mercury intoxicated industry in Brazil. Environmental Toxicology and Pharmacology, 19, 523-529.
Vetherill, G. B., \& Levitt. H. (1965). Sequential estimation of points on a psychometric function. The British Journal of Mathematical and Statistical Psychology, 48, 1-10.

Vleugels, L., van Nunen, A., Lafosse, C., Ketelaer, P., \& Vandenbussche, E. (1998). Temporal and spatial resolution in foveal vision of multiple sclerosis patients. Vision Research, 38, 2987-2997.

Wilson, H. R. (1988). Development of spatiotemporal mechanisms in infant vision. Vision Research, 28, 611-628.

Yuodelis, C., \& Hendrickson, A. (1986). A qualitative and quantitative analysis of the human fovea during development. Vision Research, 26, 847-855.
Recebido em 06.12.07

Primeira decisão editorial em 10.09.08

Versão final em 11.03.09

Aceito em 17.03.09 